\title{
Systematic and Meaningful Keyword Finding Over Encipher Data in Steam
}

\author{
CH Padma, D.Rammohanreddy
}

\begin{abstract}
Cloud computer has actually created a great deal of interest within the evaluation area over the last few years for its several blessings, nevertheless has but also raise security and also privacy concerns. The storage and gain access to of personal documents are recognized collectively of the main problems within the area. particularly, numerous scientists explored services to examine encrypted records continue remote cloud web servers. whereas numerous schemes are planned to perform conjunctive key words search, much less interest has been kept in mind on extra specialist keeping an eye out methods. throughout this paper, we tend to gift an expression search strategy supported Blossom filters that's significantly quicker than existing options, with comparable or higher storage as well as interaction cost. Our technique makes use of a series of n-gram filters to support the practicality. The style exhibits a trade-off between storage space and also false positive rate and also is filmable to resist inclusion-relation attacks. A design method sustained associate application's target incorrect positive price is additionally stood for.
\end{abstract}

\section{INTRODUCTION}

Recollect a cloud-based social affirmation records shape that has actually re-appropriated solitarysuccess information (PHRs) from sure social guarantee providers. The PHRs are engraved in solicitation to comply with wellbeing and safety headings like HIPAA. well known to draw in information use and moreover sharing, it's miles altogether pulling in have an correctly available record encryption (SE) plot which introduces the cloud talented system to look for over blended PHRs in mild of a genuine dread for the confirmed clients, (as an instance, remedial examiners or specialists) without locating facts approximately the key plaintext. understand that the placing we're questioning supports individual information sharing amongst various facts dealers and moreover diverse statistics customers. As crucial, SE designs within the non-public-key setting which understand that a solitary patron who appears for and recovers his/her amazingly declare amazing records, are not suitable. definitely, personal facts improving (PIR) indicates which hook up with clients to recover a selected statistics point from an facts supply which wholeheartedly stores subtleties with out uncovering the information point to the database supervisor, are what is greater not proper, due to the fact they need the records to be uninhibitedly handy. with the intention to address the catchphrase search for fear in the cloud-based

medicinal institutions information shape state of affairs, we recollect open up key encryption with watchword experiment for (PEKS) plans, that's directly prescribed in. In a PEKS plot, a ciphertext of the watchwords called "PEKS ciphertext" is joined with to a recorded PHR. To recover all the engraved PHRs having a watchword, state "Diabetes", a customer conveys a "trapdoor" related with a search for hobby on the catchphrase "Diabetes mellitus" to the cloud advantage service provider, which selections all of the joined PHRs containing the catchphrase "Diabetes mellitus" and furthermore returns them to the customer whilst with out engrossing the valuable PHRs. Regardless, the technique in nicely as other existing PEKS methodologies which improve basically help stability request. building up going crosswise over thing and moreover meta keywords 1 can be used for conjunctive watchword appearance. anyways, the method thinking about built up union factor discharges extra statistics to the cloud server past the held off very last products of the conjunctive hobby, while the technique using meta catchphrases require $2 \mathrm{~m}$ meta signs and symptoms to require all the attainable conjunctive hobby for $\mathrm{m}$ catchphrases. So in regards to determine the above insufficiencies in conjunctive catchword look, plans, as an instance, those in had been superior in humans with every reputedly insignificant element mulled over essential setting. preferably, in the down to earth applications, appearance predicates (i.e., sport-plans) should be vital with a convincing goal that they may be given as blend, disjunction or any form of Boolean formulas 2 of catchwords. in the above cloud-based accommodating associations structure, to reveal the organization amongst diabetic problems and age or then again weight, a convenient professional may additionally provide an interest question a methods shape (i.e., predicate) (" sickness = Diabetes Mellitus" in addition, (" Age = 30" OR "Weight $=150$-two hundred")). SE structures persevering with expressive catchphrase arrive at structures were exhibited in. rather, the direction of movement in has extremely widening diverse nature, at the same time as the strategies in rely on the inefficient bilinear mixing over composite-kind out gatherings. As institutions and moreover humans grasp cloud present day innovations, numerous wound up being upright to the extreme variables to reflect onconsideration on stressing health and security just as near home safety of getting to individual just as treatment over the net. specially, 
the ongoing and furthermore proceeded with gaining knowledge of breaks characteristic the need for greater relaxed disbursed garage frameworks at the same time as it is usually joined that cryptography is crucial, cloud vendors with the aid of and massive do the cryptography and moreover keep the character mysteries rather than the records home proprietors. this is, the cloud will absolutely take a look at any form of information it wanted, giving no near home protection to its clients.

\section{Relevant Work:}

After Bonehet al. [1] started out the assessment of open key safety with catchphrase scan for PEKS), a pair PEKS headways had been advanced utilising clean techniques or considering numerous problems [2], [3], [4], [5], [6], [7], [8], [9], [10], [11], [12], [13], [14], [15] They plan to realise 2 cruces in PEKS: (1) precisely a way to make PEKS ensured towards expelled atchword glossary approximating strikes; and furthermore (2) precisely a way to achieve giant searching predicates in PEKS. To the diploma the separated slogan word list you decide approximately ambushes, which necessitates that no adversary (checking the cloud attempting to discover server) can absorb signs from an utilized trapdoor, to the fine of our seeing, this kind of wellbeing and safety concept is tough to be developed in people normally essential putting [16] As for sizeable pursue, there are absolutely couple of labor in PEKS2 [8], [5], [6], [7] alas, the headway in [5] relies upon the begin of indoor point predicate encryption [8], just as the enhancements in [2], [6], [7] are worked from the pairings in composite-arrange joyful celebration. along those strains, they're no longer effectively convincing to be recounted within the utilitarian globe [17], [18] similarly, the degree of catchphrases conceded those open strategies are predefined within the framework layout plan. We balance out our arrangement with different catchphrase look for plots in desk 1. it's far clean to peer that stood out from the cutting-edge ones, our improvement make a decent night time out in that it certifies countless catchphrases, underpins expressive get entry to systems, simply as is worked within the high-arrange social affairs. private-key Searchable Encryption. In a non-public-key SE placing, a patron trades its non-public information to a remote data supply also, maintains the records person from the remote information source chief. non-public-key SE permits the patron to recoup each one of the facts which includes a specific catchphrase from the remote database [19], [20], [21] For any state of affairs, because the name proposes, private-key SE approaches virtually follow to situations wherein statistics owners and moreover facts customers absolutely trusted one another. individual data Retrieval. concerning open statistics source, as an instance, inventory articulations, wherein the purchaser is stressed of it and wishes to look for some information issue without providing to the information source director which factor it is, private subtleties recovery (PIR) [22], [23], [24] practices have been brought, which allow a client to recoup information from an open database with some distance humbler correspondence by then basically downloading and introduce the entire database. before lengthy, in our specific state of affairs, the data supply isn't direct presented, the facts isn't open, so the PIR publications of action can not be related.

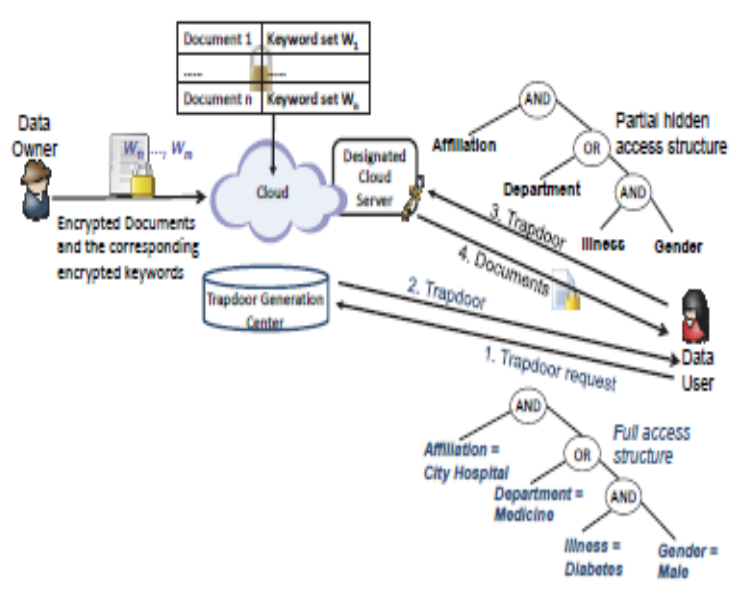

The layout of our catchphrases seek framework is gotten Fig., that's constructed from 4 elements: a confided in snare entryway age recognition who distributes the framework degree simply as holds an ace personal stunt simply as is responsible for trapdoor age for the framework, records owners who re-appropriate encoded facts to an open cloud, facts clients who're fortunate to appearance simply as get admission to scrambled information, absolutely as a checked cloud server that executes the watchword test sports activities for statistics customers. To permit the cloud server to look over ciphertexts, the data proprietors embody every encoded file with scrambled keywords4. An information consumer troubles a trapdoor request via conveying a watchword get admission to shape to the trapdoor age consciousness which produces and furthermore restores a trapdoor evaluating to the get entry to device. We count on that the trapdoor age awareness has a one in every of a type check device to approve every records client and after that discharge them the coordinating buying a trapdoor, the facts purchaser conveys the trapdoor and moreover the proportionate incomplete undercover get entry to shape (i.e., the get admission to system without are seeking query esteems) to the assigned cloud server. The final does the trying out responsibilities among every ciphertext and furthermore the trapdoor the use of its non-public mystery, just as advances the coordinating ciphertexts to the statistics purchaser. As talked about earlier than, a ciphertext made thru an facts proprietor contains sections: the encoded report created the use of a report encryption plan and furthermore the scrambled watchwords added the usage of our SE conspire. From proper now on, we sincerely ponder the remaining phase of the scrambled report, and furthermore push aside the fundamental element considering that it is out of the degree of this paper. In define, the style destinations of our expressive SE plan are fourfold. The prescribed framework ought to preserve catchphrase get to structures communicated in any form of Boolean recipe with in addition to well as doors. The encouraged association 
must be certainly powerful regarding computation, correspondence and extra room for precious applications. at the beginning, a ciphertext with out its comparing trapdoors need to no longer discover any kind of insights approximately the hunt question esteems it consists of to the cloud server and furthermore pariahs. $2 \mathrm{~d}$, a trapdoor have to not hole subtleties on catchphrase expression esteems to an out of doors aggressors with out the selective thriller of the stamped cloud net server. We get this concept of safety for the SE framework as far as semantic properly-being and protection to make sure that encoded facts does now not locate any shape of records concerning the watchwords worths, which we call "particular in understand potential as opposed to picked catchphrase set strike (cautious IND-CKA security)" (See Appendix A). The health and protection of the prescribed plan want to be officially affirmed beneath the normal shape in place of the informal assessment. We anticipate that the trapdoor age interest is a confided in element. The cloud server is thought to be "honest butcurious", i.e., it's going to sincerely preserve rapid to the with an character information from the information placed away in the cloud. information proprietors are perception to really preserve their facts, on the identical time as facts customers aren't relied on, certainly as they may be able to likewise scheme with a risky cloud net server as a manner to exchange take a look at system to approve records humans in advance than discharging trapdoors to people. furthermore, we assume that every one foes have simply confined computational restriction, so that they cannot ruin the formerly referred to extreme problems.

We implement our course of action in Appeal [25], which is a structure made to involve clever prototyping of cryptographic plans as well as conventions. Due to the Python programming language, Appeal reels in one to perform a cryptographic plan with not a great deal of lines of code, basically reducing renovation time. Then, computationally elevated intelligent tasks are completed with surrounding modules, so the overhead in point of view of Python in Appeal is under 1\%. Considering that all Charm designs are arranged under the unequal parties, our enhancement is transformed to the uneven setup before the use. That is, 3 celebrations $\mathrm{G}, \wedge \mathrm{G}$ as well as $\mathrm{G} 1$ are used also, the planning $\wedge$ e is a restriction from $G_{-} \wedge{ }^{\wedge}$ to $G 1$. Notice that it has actually been conveyed because the concerns and also the protection confirmations can be altered over to the adrift setup traditionally. The computational expenses of the Configuration and sKeyGen tallies are prompt, as well as we base on the computational expenditures of the Trapdoor, Encrypt and also Examination calculations. In our evaluations, a lot of watchwords is made, of which each catchphrase has a nonexclusive name, as an example, "Health issues", "Position", "Association" as well as a countersign respect, for example, "Diabetic issues", "Master", and also "City Health center". For coordinate use, we make use of digits to mean catch phrase worths, e.g., a catchphrase as "Illness $=6 "$ is handed down by "Sickness = Diabetes approach yet it is furthermore intrigued to end up familiar discover individual statistics of various gala's. We receive that the trusted trapdoor age attention is prepared with an

\section{EXPERIMENTAL REVIEW}

mellitus". Along these lines, we make an eccentric collection of signs consisting of 10 to 50 catchphrases, really as use them to encode 5,000 reminiscences. We as of now expel the watchword worths in the ciphertexts with the real objective that they incorporate of basically critical names of symptoms like "clinical problems", "function", as showed in our robust improve. beginning there, we discretionarily pick out 2 to ten symptoms to design a flighty get entry to structure. The approach of watchwords in a searching out interest is robotically under 10 , as showed up the scanning for solicitation logs of net crawlers [26] The treatment tree is joined with the bona fide purpose that for any type of inside accentuation factor the refinement at the inner point collection of its left branch and that of its gain department is beneath 2 . We make 50 various access technique bushes, 10 for every single thrilling collection of catchphrases, and make a trapdoor for each machine tree.We additionally dispose of the signal appreciate records from the trapdoors. So the method tree in eight. For the unequivocal information on attraction, if its every apparently insignificant element the nearly identical to you propose [25] keep as a incredible priority that because it has been plainly showed up in that the plentifulness of strategies in composite-orchestrate gatherings is on a totally fundamental stage drastically extra terrible than that of testimonies in excessive-type out get-togethers, we may not realise the ones systems in composite-sort out social affairs.moreover, the modern interpretation appeal does not manual cryptographic plans in compositeorder get-togethers. moreover, we've a have a look at the ciphertexts. For a blend of the catchphrase names in the ciphertext that satisfies the phase affiliation of the trapdoor, our catchphrase experiment for tale runs the check test to more make certain whether or not or not it is a legitimate suit the ones checks are created greater terrific than four top notch elliptic curves: SS512, MNT159, MNT201 and MNT224, of which SS512 is a supersingular elliptic twist with the bilinear expecting it being symmetric type 1 blending, without a doubt due to the fact the pairings on the other 3 turns are lopsided kind 3 pairings. those four turns gives guarantee estimations of eighty-tad, 80-bit, 100-piece just as 112-piece, self-rulingly. The figuring time for the exponentiation and readiness estimation over the 4 twists are taped.

\section{FINAL THOUGHTS \& RESULTS}

So as to allow a cloud web server to view on clambered information without taking in the fundamental plaintexts in the publickey setup, Boneh proposed a cryptographic crude called open vital file encryption with catchphrase look for (PEKS). From that point ahead, considering numerous requirements virtually speaking, e.g., communication overhead, looking for standards as well as safety enhancement, different sorts of obtainable encryption structures have actually been advanced. Nonetheless, there exist simply a couple of open vital accessible encryption structures that aid expressive countersign look approaches, and also they are completely worked from the wasteful 
composite-arrange bunches. In this paper, we focused on the framework and also evaluation of open key available security frameworks in the prime-arrange numbers that can be used to check out various watchwords in expressive looking for dishes. Due to a comprehensive cosmos key-approach quality based encryption conspire offered, we introduced an expressive obtainable encryption framework in the prime order accumulation which underpins meaningful access frameworks communicated in any kind of monotonic Boolean dishes. In addition, we demonstrated its protection in the conventional model, as well as broke down its performance making use of PC recreations.

\section{REFERENCES}

1. D. Boneh, G. D. Crescenzo, R. Ostrovsky, and G. Persiano, "Open critical protection with catchphrase are seeking for," early in Cryptology-EUROCRYPT 2004, worldwide meeting at the idea and Applications of Cryptographic techniques, Interlaken, Switzerland, May2-6, 2004, method, ser. speak Notes in computer technological know-how, vol.3027. Springer, 2004, pp. 506- - 522.

2. J. Lai, X. Zhou, R. H. Deng, Y. Li, sincerely as adequate. Chen, "Expressivesearch on encoded records," in 8th ACM Seminar on facts, laptop and moreover Communications safety, ASIA CCS 'thirteen, Hangzhou, China - may additionally 08 - 10, 2013. ACM, 2013, pp 243- - 252.

3. Y. H. Hwang certainly as P. J. Lee, "Open critical encryption with conjunctivekeyword are trying to find and its extension to a multi-consumer framework," inPairing-primarily based Cryptography - Matching 2007, First global meeting, Tokyo, Japan, July 2-4, 2007, court cases, ser. talk Notesin pc innovation, vol. 4575. Springer, 2007, pp. 2- - 22.

4. B. Zhang and furthermore F. Zhang, "A solid open fundamental encryption withconjunctive-subset watchwords search," J. gadget and pc applications Experimental results for the test recipe over numerous elliptic bends.

5. D. Boneh genuinely as B. Oceans, "Conjunctive, detail, and range inquiries on scrambled records," in concept of Cryptography, fourth idea of Cryptography assembly, TCC 2007, Amsterdam, The Netherlands February 21-24, 2007, way, ser. talk Notes in pc era, vol. 4392. Springer, 2007, pp. 535- - 554.

6. Z. Lv, C. Hong, M. Zhang, and moreover D. Feng, "Expressive and sheltered and comfy handy encryption within the standard population essential affiliation," in information protection And safety - 17th international convention, ISC 2014, Hong Kong, China, October 12-14, 2014. strategies, ser. talk Notes in ComputerScience, vol. 8783. Springer, 2014, pp. 364- - 376.

7. J. Shi, J. Lai, Y. Li, R. H. Deng, and J. Weng, "prison watchword are searching for on encoded facts," in computer tool protection And protection - ESORICS 2014 - 19 th eu Seminar on studies observe in pc system safety, Wroclaw, Poland, September 7-eleven, 2014. techniques, detail I, ser. communicate Notesin pc science, vol. 8712. Springer, 2014, pp. 419- - 435.

8. H. S. Rhee, J. H. Park, W. Susilo, and furthermore D. H. Lee, "Upgraded on hand open key safety with checked analyzer," in method of the 2009 ACM Symposium on information, computer framework andCommunications safety, ASIACCS 2009, Sydney, Australia, March10-12, 2009. ACM, 2009, pp. 376- - 379.

9. M. Bellare, A. Boldyreva, and moreover A. O'Neill, "Deterministic and effectively handy encryption," in
Advances in Cryptology - CRYPTO 2007, 27th Annual international Cryptology Seminar, Santa Barbara, CA, u.s., August 19-23, 2007, procedure, ser. communicate Notes in computer technological know-how, vol. 4622. Springer, 2007, pp. 535- - 552.

10. C. Gu, Y. Zhu, just as H. Griddle, "strong open key encryption with watchword expression are searching for plans from pairings," in info safety and Cryptology, 1/3 SKLOIS Seminar, Inscrypt 2007, Xining, China, August 31 - September five, 2007, Revised selected Papers, ser.Lecture Notes in computer technology, vol. 4990. Springer, 2007, pp. 372- - 383.

11. J. Baek, R. Safavi-Naini, and furthermore W. Susilo, "Open fundamental document encryption with key expression seek looked yet again at," in Computational scientific research just as Its packages - ICCSA 2008 , international Seminar, Perugia, Italy, June 30 - July three, 2008, method, aspect I, ser. talk Notes in laptop generation, vol. 5072. Springer, 2008, pp. 1249- - 1259.

12. Q. Tang virtually as L. Chen, "Open key safety with enrolled catchphrase expression search," in Public Key Infrastructures, corporations and applications - 6th european Workshop, EuroPKI 2009, Pisa, Italy, September 10-11, 2009, Revised selected Papers, ser. communicate Notes in ComputerScience, vol. 6391. Springer, 2009, pp. 163- - 178.

13. M. Li, S. Yu, N. Cao, definitely as W. Lou, "popular character watchword are seeking over encoded records in dispensed computing," in 2011 international meeting on allotted Computing structures, ICDCS 2011, Minneapolis, Minnesota, americaa., June 20-24, 2011. IEEE pc tradition, 2011, pp. 383- - 392.

14. H. S. Rhee, J. H. Park, and moreover D. H. Lee, "regular structure of assigned analyzer open key file encryption with catchphrase expression search," Inf. Sci., vol. 205, pp. ninety three- - 109, 2012.

15. W. Yau, R. C. Phan, S. Heng, and moreover B. Goi, "searching for question questioning attacks on safe available open key protection plans with an assigned analyzer," Int. J. Comput. Math., vol. ninety, no. 12, pp. 2581- - 2587, 2013.

16. E. Shen, E. Shi, and moreover B. Waters, "Predicate individual safety in safety frameworks," in principle of Cryptography, 6th idea of Cryptography Seminar, TCC 2009, San Francisco, CA, u.s.A., March 15-17, 2009. systems, ser. speak Notes in computer innovation, vol. 5444. Springer, 2009, pp. 457- - 473.

17. J. Katz, A. Sahai, and moreover B. Oceans, "Predicate document encryption assisting disjunctions, polynomial conditions, and inner gadgets," J. Cryptology, vol. 26, no. 2, pp. 191- - 224, 2013.

18. D. M. Freeman, "converting blending based totally cryptosystems from composite-request gatherings to high-request gatherings," in Advances in Cryptology EUROCRYPT 2010, twenty ninth every year global Seminar at the concept and moreover packages of Cryptographic strategies, French Riviera, may additionally 30 - June three, 2010. procedures, ser. communicate Notes in pc generation, vol. 6110. Springer, 2010 , pp. forty four- - 61 .

19. O. Goldreich just as R. Ostrovsky, "Programming resistance and moreover reenactment on unconcerned rams," J. ACM, vol. 40 three, no. three, pp. 431- - 473, 1996.

20. D. X. Melody, D. Wagner, simply as A. Perrig, "affordable processes for hunts on encoded information," in 2000 IEEE Symposium on protection and furthermore 
personal privateness, Berkeley, California, usa, may also 14-17, 2000. IEEEComputer life-style, 2000, pp. 40 4- fifty five.

21. E. Goh, "secure facts," IACR Cryptology ePrint Archive, vol.2003, p. 216, 2003.

22. C. Cachin, S. Micali, sincerely as M. Stadler, "Computationally elite records restoration with polylogarithmic correspondence," in Advances in Cryptology - EUROCRYPT '99, global Seminar at the idea and alertness of Cryptographic strategies, Prague, Czech Republic, can also 2-6, 1999, continuing, ser. speak Notes inComputer clinical research, vol. 1592. Springer, 1999, pp. 402- - 414.

23. G. D. Crescenzo, T. Malkin, and R. Ostrovsky, "single statistics source person subtleties healing shows ignorant exchange," early in Cryptology - EUROCRYPT 2000, international convention on the concept and moreover software program of Cryptographic techniques, Bruges, Belgium, also can 14-18, 2000, continuing, ser. communicate Notes in computer innovation, vol. 1807. Springer, 2000, pp. 122- - 138.

24. W. Ogata just as k. Kurosawa, "Ignorant watchword expression are seeking for," J. Multifaceted nature, vol. 20, no. 2-3, pp. 356- - 371, 2004.

25. J. A. Akinyele, C. Garman, I. Miers, M. W. Pagano, M. Rushanan, M. green, and A. D. Rubin, "Bid: a shape for quick prototyping cryptosystems," J. Cryptographic format, vol. 3, no. 2, p. 111- - 128, 2013.

26. L. Yang, Q. Mei, ok. Zheng, and D. A. Hanauer, "Request $\log$ analysisof an digital nicely-being and health file web index," in Proc. of AMIA yearly Seminar, 2011, p. 915C924 